\title{
QUALIDADE FISIOLÓGICA DE SEMENTES DE MILHO SOB CONDIÇÕES DE ESTRESSE SALINO
}

\author{
José Alves Pessoa Neto ${ }^{1}$, Joelma Francisca de Moura Lima ${ }^{1}$, Fabio Mielezrski ${ }^{2}$, Soislan \\ Sousa Reis ${ }^{1}$, Maisa de Sousa Veras ${ }^{1}$
}

\begin{abstract}
${ }^{1}$ Mestrando(a) em Agronomia - Fitotecnia, Universidade Federal do Piauí, Campus Prof ${ }^{a}$ Cinobelina Elvas, Bom Jesus - PI.

${ }^{2}$ Professor Adjunto, Fitotecnia, Universidade Federal do Piauí, Campus Prof ${ }^{a}$ Cinobelina Elvas, Bom Jesus PI.
\end{abstract}

RESUMO: O milho (Zea mays L.) é cultivado em todo território nacional, tendo grande importância econômica para o país. No entanto algumas regiões brasileiras apresentam fatores limitantes como a presença de solos e água salinizados. O objetivo do trabalho foi avaliar em diferentes concentrações salinas o desempenho de quatro cultivares de milho sobre os aspectos germinativos e crescimento da planta. O delineamento experimental utilizado foi inteiramente casualizado, com quatro repetições. Os tratamentos foram instalados em esquema fatorial $5 \times 4$. O primeiro fator, salinidade, foi composto por cinco níveis de salinidade da água $\left(0 ; 0,5 ; 1,0 ; 2,0\right.$ e $\left.3,0 \mathrm{dS} \mathrm{m}^{-1}\right)$. As variáveis avaliadas foram germinação, primeira contagem da germinação, índice de velocidade de germinação, comprimento de plântulas, massa seca e massa fresca. $\mathrm{O}$ aumento da concentração salina no substrato reduziu a germinação, índice de contagem de germinação, primeira contagem de germinação e matéria seca, especialmente das cultivares P3844H e AL Bandeirantes, mostrando uma maior sensibilidade à salinidade. O cultivar 30FS3 YH destacou-se como a mais tolerante a salinidade em todas variáveis analisadas.

Palavras-chave: $\mathrm{NaCl}$. Plântulas. Zea mays L.

\section{PHYSIOLOGICAL QUALITY CORN SEED UNDER CONDITIONS OF STRESS SALINE}

ABSTRACT: Corn (Zea mays L.) is grown throughout the country, with great economic importance. However some Brazilian regions are limiting factors such as the presence of soils and saline water. The objective of this study was to evaluate different salt concentrations in four cultivars of corn on the germination and growth aspects of corn. The experimental design was completely randomized, with four replications. The treatments were installed in factorial $5 \times 4$. The first factor, salinity, was composed of five water salinity levels $\left(0,0.5,1.0,2.0\right.$ and $\left.3.0 \mathrm{dS} \mathrm{m}^{-1}\right)$. The variables evaluated were germination, first count, speed germination index, seedling length, dry seedling mass and fresh seedling mass. Increasing the salt concentration in the substrate reduced germination, speed germination index, first count and dry mass matter, especially the $\mathrm{P} 3844 \mathrm{H}$ and $\mathrm{AL}$ Bandeirantes cultivars, showing greater sensitivity to salinity. Cultivar 30FS3 YH stood out

Cultura Agronômica, Ilha Solteira, v.25, n.4, p.401-408, 2016 
as the salinity tolerant in all variables analyzed.

Key words: $\mathrm{NaCl}$. Seedlings. Zea mays L.

\section{INTRODUÇÃO}

O milho (Zea mays L.) originário da América Central é cultivado em território nacional, tendo grande importância econômica, devido às diversas formas de sua utilização, desde a alimentação humana e animal até a indústria de alta tecnologia e utilização na produção de biocombustíveis (FORNASIERI FILHO, 2007). No entanto algumas regiões brasileiras apresentam fatores limitantes, como a presença de solos e água salinizados, comum em locais de clima árido ou semiárido, ocasionando decréscimo na produção.

A concentração salina nos solos agrícolas costuma ser maior quando são irrigados, sendo motivo de grande preocupação, não só no Brasil, como no mundo. O uso de água salina na agricultura deve ser considerado como uma alternativa importante na utilização dos recursos naturais escassos. Entretanto, a qualidade da água para irrigação das regiões semiáridas apresenta grande variabilidade, tanto em termos geográficos (espacial), como ao longo do ano (sazonal). Dentre as características que determinam a qualidade da água para a irrigação, a concentração de sais solúveis ou salinidade, é um dos principais fatores limitantes ao crescimento e desenvolvimento de algumas culturas (BEZERRA et al., 2010).

A concentração de sais a níveis elevados pode levar a planta a, eventualmente, perder água para o meio externo (DIAS; BLANCO, 2010) e a sofrer com a toxidez, fato que irá interferir no crescimento e desenvolvimento vegetal. Com isto, vários processos fisiológicos e bioquímicos serão afetados, tais como respiração, fotossíntese, síntese de proteínas e metabolismo de lipídeos (DIAS; BLANCO; GONÇALVES et al., 2011). A necessidade de desenvolver cultivares mais tolerantes a salinidade vem sendo estudada nos últimos anos.

No entanto, devido a seu grande potencial produtivo e importância para o agronegócio nacional, necessita de mais estudos que viabilizem e incrementem sua produção em condições de solo e água salinos. Com isso no presente trabalho objetivou-se em avaliar diferentes concentrações salinas em quatro cultivares de milho sobre os aspectos germinativos e crescimento do milho.

\section{MATERIAL E MÉTODOS}

O trabalho foi realizado no Laboratório de Fitotecnia da Universidade Federal do Piauí- UFPI em Bom Jesus- PI. O delineamento experimental utilizado foi inteiramente casualizado, com quatro repetições. Os tratamentos foram instalados em esquema fatorial 5 $\mathrm{x}$ 4. O primeiro fator, salinidade, foi composto por cinco níveis de salinidade da água (0; 0,$5 ; 1,0 ; 2,0$ e $3,0 \mathrm{dS} \mathrm{m}^{-1}$ ). As soluções salinas foram preparadas com água potável e a concentração dos sais obtidos a partir da equação de Munns (2010). O segundo fator,

Cultura Agronômica, Ilha Solteira, v.25, n.4, p.401-408, 2016 
cultivar, foi composto por quatro níveis, que corresponderam aos cultivares $\mathrm{P} 3844 \mathrm{H}$, 30F53YH, AL-Bandeirantes e Crioula.

As variáveis avaliadas foram germinação, primeira contagem da germinação, índice de velocidade de germinação, comprimento, massa seca e massa fresca de plântulas. O teste de germinação foi conduzido com quatro subamostras de 50 sementes de cada tratamento em rolos de papel "Germitest" e armazenadas em câmera BOD regulado para manter a temperatura constante de $25 \pm 2{ }^{\circ} \mathrm{C}$; a quantidade de água adicionada foi equivalente a 2,5 vezes a massa do substrato seco visando ao umedecimento adequado e, consequentemente, a uniformização do teste. As interpretações foram efetuadas a partir do $1^{\circ}$ dia depois da semeadura, segundo os critérios estabelecidos nas Regras para Análise de Sementes (BRASIL, 2009) computando-se o índice de velocidade de germinação e a porcentagem média de germinação.

A primeira contagem foi realizada juntamente com o teste de germinação sendo o registro da percentagem de plântulas normais verificado ao quarto dia após a instalação do teste (BRASIL, 2009). O comprimento da raiz primária e da parte aérea foi feito de acordo com a metodologia de Nakagawa (1999), utilizadas quatro repetições de dez sementes, dispostas sobre três folhas de papel germitest, umedecidas com a concentração na quantidade equivalente a 2,5 vezes seu peso seco. As sementes foram alinhadas longitudinalmente na superfície e mantidas em câmera BOD a uma temperatura constante de 25 $\pm 2{ }^{\circ} \mathrm{C}$. Após sete dias da instalação do teste, avaliou-se o comprimento das raízes e da parte aérea das plântulas, com auxílio de paquímetro graduado, obtendo-se os valores em mm planta $^{-1}$.

Avaliou-se a massa fresca das plântulas obtidas no sétimo dia de contagem de germinação, sendo pesadas 10 plântulas por repetição. Em seguida, as plântulas foram colocadas para secar em estufa de circulação de ar forçada a $65^{\circ} \mathrm{C}$ até obter peso constante da massa seca, seguindo a metodologia de Nakagawa (1999).

Os dados foram submetidos à análise de variância e as médias foram comparadas pelo teste $\mathrm{F}$ de Snedecor, a 5\% de probabilidade de erro. Em seguida foi feito o desdobramento da interação para o efeito de cultivares em cada nível de estresse salino. O software utilizado para o processamento analítico dos dados foi o $\mathrm{R}$ versão 3.2.0.

\section{RESULTADOS E DISCUSSÃO}

Observou-se efeito significativo na cultivar e salinidade para índice de velocidade de germinação $(p<0,01)$ e porcentagem de germinação, primeira contagem de germinação e matéria seca $(\mathrm{p}<0,05)$ (Tabela 1$)$.

Cultura Agronômica, Ilha Solteira, v.25, n.4, p.401-408, 2016 
Tabela 1. Resumo da análise de variância para os caracteres porcentagem de germinação (GERM), Índice de Velocidade de Germinação (IVG), Primeira Contagem (PCON), Matéria fresca (MF), Matéria Seca (MS) e Comprimento de Plântulas (COMP) avaliadas em cultivares de milho submetido a cinco concentrações salinas

\begin{tabular}{llllllll}
\hline FV & \multirow{6}{*}{ GL } & \multicolumn{7}{c}{ QM } \\
\cline { 3 - 8 } & & \multicolumn{1}{c}{ GERM } & \multicolumn{1}{c}{ IVG } & \multicolumn{1}{c}{ PCON } & \multicolumn{1}{c}{ MF } & \multicolumn{1}{c}{ MS } & COMP \\
\hline Sal & 4 & $52,7^{\text {s }}$ & $58,5^{* *}$ & $45,9^{*}$ & $19318252^{\text {ns }}$ & $0,020^{*}$ & $10,8^{* *}$ \\
Cult & 3 & $10780^{* *}$ & $7946,6^{* *}$ & $11530,9^{* *}$ & $21457776^{\text {ns }}$ & $0,366^{* *}$ & $13,8^{* *}$ \\
Sal x Cult & 12 & $58,3^{*}$ & $29,8^{* *}$ & $37,2^{*}$ & $15466667^{\text {ns }}$ & $0,003^{*}$ & $1,6^{\text {ns }}$ \\
Erro & 60 & 26,4 & 9,4 & 15,1 & 17947290 & 0,00264 & 1,0 \\
\hline CV $(\%)$ & & 6,58 & 5,29 & 4,72 & 82,46 & 8,35 & 12,07
\end{tabular}

FV - Fontes de variação; GL - graus de liberdade; QM - Quadrados médios;; CV(\%) - Coeficiente de variação experimental. * e $* *$ Significativo a 5 e $1 \%$ de probabilidade pelo teste $\mathrm{F}$ de Snedecor; ${ }^{\text {ns }}$ Não significativo.

As sementes das cultivares de milho submetidas às concentrações de $\mathrm{NaCl}$ mostrou-se que o potencial germinativo não foi afetado de acordo com a concentração de $\mathrm{NaCl}$, mas observou-se a interação entre a cultivar e a concentração de $\mathrm{NaCl}$ foi significativa a $5 \%$ de probabilidade (Tabela 1). Isso significa que os cultivares tiveram comportamento diferente em relação às concentrações salinas. O cultivar Crioula e 30F53YH apresentaram maior porcentagem de germinação quando submetida em todas as concentrações salinas estudadas em relação à $\mathrm{P} 3844 \mathrm{H}$ e a $\mathrm{AL}$ - Bandeirante, demostrando que ambos apresentam tolerância ao estresse salino (Tabela 2).

Resultados semelhantes foram encontrados em sementes de gliricidia por Farias et al. (2009) e Carvalho e Kazama (2011) em sementes de pepino onde também ocorre decréscimo da germinação de acordo com o acréscimo de $\mathrm{NaCl}$. Este efeito deletério pode ser referente à elevada concentração de solutos na solução, provocando um déficit hídrico pela redução do potencial osmótico e ação de alguns íons, decorrente dos elevados teores de $\mathrm{Na}+$ e $\mathrm{Cl}-$, e da alterada relação $\mathrm{K}+/ \mathrm{Na}+$ e outros nutrientes (WILLADINO; CAMARA, 2010).

Comparando o efeito do estresse salino com os cultivares, verificou-se que o índice de velocidade de germinação (IVG) foi a variável mais afetada, à medida que ocorre o aumento a concentração de $\mathrm{NaCl}$, reduziu o índice de velocidade de germinação, exceto para cultivar 30F53 YH que demostrou ser tolerante com o aumento $\mathrm{NaCl}$, atingindo maior velocidade de germinação em salinidade $1,0 \mathrm{dS} \mathrm{m}{ }^{-1}$ (Tabela 2).

Tal resultado corrobora com Souza et al (2010) em sementes de pinhão manso, verificando que quanto maior a concentração salina, menor velocidade de germinação. $\mathrm{O}$ índice de velocidade de germinação é um dos principais parâmetros a ser avaliado em um teste de germinação, pois segundo Lima et al (2005) quanto mais rapidamente à semente germina, maior é o seu vigor.

Cultura Agronômica, Ilha Solteira, v.25, n.4, p.401-408, 2016 
Tabela 2. Desdobramento da interação para o efeito de cultivares (CULT) em cada nível de estresse salino

\begin{tabular}{|c|c|c|c|c|c|c|c|c|}
\hline \multirow{2}{*}{ CULT } & \multicolumn{3}{|c|}{$0 \mathrm{dS} \mathrm{m}^{-1}$} & \multirow[b]{2}{*}{ MS } & \multicolumn{4}{|c|}{$0,5 \mathrm{dS} \mathrm{m}^{-1}$} \\
\hline & GERM & IVG & PCON & & GERM & IVG & PCON & MS \\
\hline P3844H & $52,5 \mathrm{c}$ & $33,60 \mathrm{a}$ & $53,5 b$ & $0,50 \mathrm{c}$ & $43,00 \mathrm{c}$ & $27,49 \mathrm{c}$ & $48,5 b$ & $0,45 c$ \\
\hline $30 \mathrm{~F} 53 \mathrm{YH}$ & $87 b$ & $70,53 a$ & $90,5 a$ & $0,89 a$ & $87,5 \mathrm{ab}$ & $71,48 \mathrm{a}$ & $92,00 \mathrm{a}$ & $0,77 \mathrm{a}$ \\
\hline Bandeirante & $84,5 b$ & $71,18 \mathrm{a}$ & $94,5 \mathrm{a}$ & $0,62 b$ & $84,5 b$ & $62,52 b$ & $92,00 \mathrm{a}$ & $0,62 b$ \\
\hline \multirow[t]{2}{*}{ Crioula } & $98,5 \mathrm{a}$ & $67,03 b$ & $97,00 \mathrm{a}$ & $0,66 b$ & $97,00 \mathrm{a}$ & $62,12 b$ & $98,5 a$ & $0,67 b$ \\
\hline & \multicolumn{4}{|l|}{$1 \mathrm{dS} \mathrm{m}^{-1}$} & \multicolumn{4}{|c|}{$2 \mathrm{dS} \mathrm{m}^{-1}$} \\
\hline P3844H & $42,00 \mathrm{~b}$ & $28,23 \mathrm{~d}$ & $43,5 b$ & $0,46 \mathrm{c}$ & $42,00 \mathrm{c}$ & $28,06 \mathrm{c}$ & $43,5 \mathrm{c}$ & $0,45 \mathrm{c}$ \\
\hline $30 \mathrm{~F} 53 \mathrm{YH}$ & $95,00 \mathrm{a}$ & $75,4 a$ & $97,5 \mathrm{a}$ & $0,82 \mathrm{a}$ & $89,5 \mathrm{ab}$ & $72,18 \mathrm{a}$ & $91,00 \mathrm{ab}$ & $0,81 \mathrm{a}$ \\
\hline Bandeirante & $85,5 a$ & $69,49 b$ & $94,00 \mathrm{a}$ & $0,55 \mathrm{bc}$ & $82,00 \mathrm{~b}$ & $66,81 \mathrm{a}$ & $88,00 \mathrm{~b}$ & $0,55 b$ \\
\hline \multirow[t]{2}{*}{ Crioula } & $93,5 a$ & $60,84 \mathrm{c}$ & $98,00 \mathrm{a}$ & $0,58 b$ & $92,5 \mathrm{a}$ & $57,51 \mathrm{~b}$ & $95,5 \mathrm{a}$ & $0,52 b c$ \\
\hline & \multicolumn{4}{|l|}{$3 \mathrm{dS} \mathrm{m}^{-1}$} & & & & \\
\hline P3844H & $39,00 \mathrm{~b}$ & $25,72 \mathrm{c}$ & $43,5 b$ & $0,49 \mathrm{~b}$ & & & & \\
\hline 30F53YH & $87,5 \mathrm{a}$ & $72,65 a$ & $94,5 \mathrm{a}$ & $0,65 \mathrm{a}$ & & & & \\
\hline Bandeirante & $89,00 \mathrm{a}$ & $70,91 \mathrm{a}$ & $96,00 \mathrm{a}$ & $0,62 \mathrm{a}$ & & & & \\
\hline Crioula & $89,5 a$ & $62,80 \mathrm{~b}$ & $96,5 a$ & $0,57 \mathrm{ab}$ & & & & \\
\hline
\end{tabular}

Médias seguidas por letras distintas nas colunas, diferem entre si, pelo teste de tukey a 5\% de probabilidade.

O efeito estresse salino promoveu o decréscimo na primeira contagem de germinação no cultivar P3844H de milho conforme o aumento das concentrações $\mathrm{NaCl}$ (Tabela 1), para os outros três cultivares embora se tenha observado interação entre cultivares e níveis salinos não se observaram variações bruscas na primeira contagem de germinação com o aumento das concentrações salinas o que pode ser verificado na Tabela 2. De forma geral, qualquer planta tem seu desenvolvimento normal prejudicado pela presença de elevadas concentrações de sais no solo ou na água de irrigação, o que diferencia é que algumas espécies têm tolerância a níveis maiores de salinidade, enquanto outras são mais sensíveis (BARRETO, 2010).

Com o aumento da salinidade, a germinação e o desenvolvimento das plântulas foi diretamente comprometido, à medida que as sementes absorvem água salina de alta solubilidade, como é o caso do $\mathrm{NaCl}$, estes se tornam tóxicos e por consequência a ocasionam distúrbios fisiológicos, redução de potencial hídrico e decréscimo no potencial de germinação, podendo originar plântulas com menor porte (CARVALHO; KAZAMA, 2011).

A massa seca total proporcionou resultados significativos pelos níveis salinos e cultivares apresentando também interação entre ambos os fatores avaliados. De forma geral, a biomassa das plântulas demonstrou um crescimento e desenvolvimento proporcional de seus órgãos, no entanto ocorreu uma leve redução de matéria seca a partir da elevação da salinidade 0,5 dS.m- . Segundo Lima et al (2005) estudando sementes de arroz, observaram que relação a matéria seca da parte aérea e raízes decresce com aumento na concentração salina.

Cultura Agronômica, Ilha Solteira, v.25, n.4, p.401-408, 2016 
A redução da matéria seca das plântulas de milho com o aumento da concentração de $\mathrm{NaCl}$ pode está relacionada com o déficit hídrico causado pelas altas concentrações salinas. O ingresso de sais na solução do solo causa déficit hídrico nas folhas e inibe o crescimento e o metabolismo do vegetal (TAIZ; ZEIGER, 2013).

Os prejuízos causados pelo estresse salino representados na matéria seca mostram que a salinidade, além de diminuir a produção de biomassa, também pode alterar a partição de fotoassimilados nas diferentes partes das plantas (SILVA et al, 2003).

Um alto nível salino diminui a taxa de assimilação metabólica, a atividade de enzimas responsáveis pela respiração e fotossíntese, minimizando a obtenção de energia para o crescimento e diferenciação das células em tecidos, ocorrendo consequentemente a redução do alongamento do eixo embrionário e a produção de massa seca (TAIZ; ZEIGER, 2013).

\section{CONCLUSÃO}

O aumento da concentração salina no substrato reduziu a germinação total, índice de velocidade de germinação, primeira contagem de germinação e matéria seca, especialmente dos cultivares $\mathrm{P} 3844 \mathrm{H}$ e AL-Bandeirantes, mostrando uma maior sensibilidade à salinidade.

O cultivar 30FS3 YH destacou-se como o mais tolerante a salinidade em todas variáveis analisadas.

\section{REFERÊNCIAS BIBLIOGRÁFICAS}

BARRETO, H. B. F.; FREITAS, R. M. O. D.; OLIVEIRA, L. A. D. A.; ARAUJO, J. A. D. M.; COSTA, E. M. D. Efeito da irrigação com água salina na germinação de sementes de sábia (Mimosa caesalpiniifolia Benth). Revista Verde, Mossoró, v. 5, n. 3, p.125-130, 2010 .

BEZERRA, A. K. P.; LACERDA, C. F.; HERNANDEZ, F. F. F.; SILVA, F. B.; GHEYI, H. R. Rotação cultural feijão caupi/milho utilizando-se águas de salinidades diferentes. Ciência Rural, Santa Maria, v. 40, n. 5, p.1075-1082, 2010.

BRASIL. Ministério da Agricultura e Reforma Agrária. Secretaria Nacional de defesa Agropecuária. Regras para análise de sementes. Brasília: Mapa/ACS, 2009. 395 p.

CARVALHO, L. C.; KAZAMA, E. H. Efeito da salinidade de cloreto de potássio ( $\mathrm{KCl})$ na germinação de sementes e crescimento de plântulas de pepino (Cucumis sativus L.). Enciclopédia Biosfera, Centro Científico Conhecer, Goiânia, v. 7, n. 1, p.429-435, 2011.

DIAS, N. S.; BLANCO, F. F. Efeitos dos sais no solo e na planta. In: GHEYI, H. R.; DIAS, N. S.; LACERDA, C. F. (Ed.). Manejo da salinidade na agricultura: estudos básicos e aplicados. Fortaleza: Instituto Nacional de Ciência e Tecnologia em Salinidade, 2010. cap. 9, p. 127-141,

Cultura Agronômica, Ilha Solteira, v.25, n.4, p.401-408, 2016 
FARIAS, S. G. G.; FREIRE, A. L. O.; SANTOS, D. R.; BAKKE, I. A.; SILVA, R. B. Efeitos dos estresses hídrico e salino na germinação de sementes de gliricidia [Gliricidia sepium (JACQ.) STEUD.]. Revista Caatinga, Mossoró, v. 22, n. 4, p.152-157, 2009.

FORNASIERI FILHO, D. Manual da cultura do milho. 1. ed. Jaboticabal: FUNEP, 2007. $576 \mathrm{p}$.

GONÇALVES, I. V. C.; FREIRE, M. B. G. S.; SOUZA, E. R.; FREIRE, F. J. Alterações químicas de um neossolo flúvico irrigado com águas salinas. Revista Ciência Agronômica, Fortaleza, v. 42, n. 3, p.589-596, 2011.

LIMA, M. G. S.; LOPES, N. F.; MORAES, D. M.; ABREU, C. M. Qualidade fisiológica de sementes de arroz submetidas a estresse salino. Revista Brasileira de Sementes, Londrina, v. 27, n. 1, p.54-61, 2005.

MUNNS, R. Osmotic stress treatments. In: Prometheus Wiki, Version 1, 2010. Disponível em: < http://www.publish.csiro.au/prometheuswiki >. Acesso em: 7 set., 2015.

NAKAGAWA, J. Testes de vigor baseados na avaliação de plântulas. In: KRZYZANOWSKI, F.C.; VIEIRA, R.D.; FRANÇA-NETO, J.B. (Ed.). Vigor de sementes: conceitos e testes. Londrina: ABRATES, 1999. p.2:1- 2:21.

R CORE TEAM. R: A language and environment for statistical computing. Vienna: R Foundation for Statistical Computing 2015. Disponível em: <https://www.R-project.org/>. Acesso em: 15 set., 2015.

SILVA J. V.; LACERDA, C. F.; COSTA, P. H. A.; ENÉAS-FILHO, J.; GOMES-FILHO, E.; PRISCO, J. T. Physiological responses of $\mathrm{NaCl}$ stressed cowpea plants grown in nutrient solution supplemented with $\mathrm{CaCl}$ 2. Brazilian Journal of Plant Physiology, Campinas, v. 15, n. 2, p.99-105, 2003.

SIVRITEPE, N. H. O.; SIVRITEPE, A. E. The effect of $\mathrm{NaCl}$ priming on salt tolerance in melon seedling grown under saline conditions. Scientae Horticulturae, Amesterdã, v. 1, n. 97, p.229-237, 2003.

SOUZA, Y. A.; PEREIRA, A. L.; SILVA, F. S. S.; REIS, R. C. R.; EVANGELISTA, M. R. V.; CASTRO, R. D.; DANTAS, B. F. Efeito da salinidade na germinação de sementes e no crescimento inicial de mudas de pinhão-manso. Revista Brasileira de Sementes, Londrina, v. 32, n. 2, p.83-92, 2010.

TAIZ, L.; ZEIGER, E. Fisiologia vegetal. 5. ed. Porto Alegre: Artmed, 2013. 918 p.

WILLADINO, L.; CAMARA, T. R. Tolerância das plantas à salinidade: aspectos fisiológicos e bioquímicos. Enciclopédia Biosfera: Centro Científico Conhecer, Goiânia, v. 6, n. 11,_p.01-23, 2010.

Cultura Agronômica, Ilha Solteira, v.25, n.4, p.401-408, 2016 
